\title{
A Novel Zero-Voltage-Transition PWM Cúk Power Factor Corrector
}

\author{
Ching-Jung Tseng Chern-Lin Chen \\ Power Electronics Laboratory \\ Department of Electrical Engineering \\ National Taiwan University \\ Taipei, Taiwan
}

\begin{abstract}
A novel zero-voltage-transition (ZVT) PWM Cúk power factor corrector (PFC) is proposed to achieve unity power factor under zero-voltage-switching (ZVS) operations. In the proposed ZVT PWM Cúk PFC, not only the power switch but also the power diode commutate under ZVS. The proposed ZVT topology has the shortest ZVT time and thus the shortest minimum duty cycle compared with other ZVT topologies. The resonant inductor can be discharged regardless of the state of the main switch. Extremely short ZVT time and robust discharge of resonant inductor make the proposed ZVT topology well qualified for variable duty and high switching frequency applications. Analytical studies on the proposed ZVT PWM Cúk PFC are presented in detail. A $400 \mathrm{~W}, 50 \mathrm{kHz}$ prototype has been built in the laboratory to experimentally verify the analysis.
\end{abstract}

\section{INTRODUCTION}

To satisfy the forthcoming harmonic standards such as IEC 1000-3-2, power factor correctors (PFC's) serving as front-end converters have been attracting more and more attention. Among various PFC topologies, the Cúk PFC topology is deemed desirable for its continuous input and output currents, ripple-free input current, small output filter and wide output voltage range [1-2]. However, the switch utilization factor of the Cúk topology is much lower than other topologies, such as the buck topology and the boost topology [3]. In other words, the power handling capability requirements of the semiconductor devices of a Cúk PFC are much higher than those of a buck PFC or a boost PFC with same output power. Reduction of switching losses and EMI noises are especially important to a Cúk PFC.

Pulse-width-modulated (PWM) technique has been widely used on dc-dc converters and PFC's in industrial applications. It is praised for the high power capability and ease of control. To achieve higher power density and faster transient response, PWM converters should operate under higher switching frequency. However, as the switching frequency increases, so do the switching losses and EMI noises. High switching losses reduce the power handling capability and serious EMI noises interfere the control of PWM converters. Several kinds of soft switching techniques, such as passive snubbers [4] and resonant converters [5], have been proposed recent years to reduce switching losses and EMI noises. The zero-voltage-transition (ZVT) PWM techniques are deemed the most commendable ones among them [6-11]. One auxiliary switch and other passive components are used to form a shunt resonant ZVT cell in a ZVT PWM converter. Switching losses and EMI noises are reduced because the shunt resonant ZVT cell works during switching transient to perform zero-voltage-switching (ZVS) or zero-current-switching (ZCS). Merits of both the conventional PWM technique and the resonant technique are maintained in ZVT PWM converters

A novel ZVT PWM Cúk PFC is presented in this paper by applying the proposed ZVT PWM topology to a Cúk PFC to effectively reduce the switching losses and EMI noises. In the proposed circuit, not only the power switch but also the power diode commutate under ZVS. The proposed ZVT PWM technique use one switch, one diode, one capacitor and one inductor to form a shunt resonant ZVT cell. Without additional component compared with the conventional ZV'T technique introduced in [6], the proposed ZVT technique dramatically improves the drawbacks of the conventional one through robuster discharge of resonant inductor and shorter ZVT time. Theoretical analysis and design procedures of the ZVT PWM Cúk PFC are studied in depth. Experimental waveforms of a $400 \mathrm{~W}$ prototype built in the laboratory are used to verify the analysis.

\section{THE ZVT PWM CÚK PFC}

\section{A. Circuit Operation Analysis}

Shown in Fig. 1 is the proposed ZVT PWM Cuk PFC. It is the combination of a conventional PWM Cúk PFC and the shunt resonant ZVT cell, which is encircled by dotted lines. The proposed shunt resonant ZVT cell consists of a resonant inductor $\mathrm{Lr}$, a resonant capacitor $\mathrm{Cr}$, an auxiliary switch $\mathrm{S}_{2}$ and an auxiliary diode $\mathrm{D} 2$. The resonant capacitor $\mathrm{C}_{\mathrm{r}}$ also incorporates the output capacitance of the power switch and the power diode. Body diodes Ds1B of the main switch $\mathrm{S}_{1}$ is also utilized in this converter 


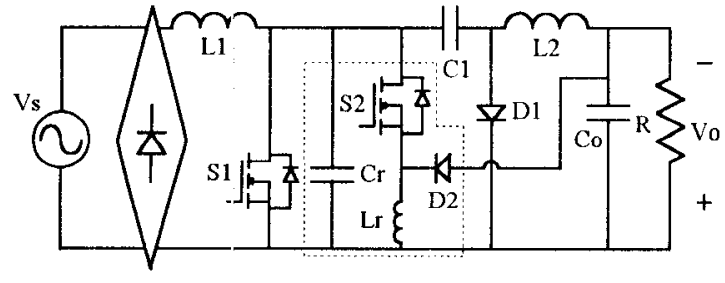

Fig. 1. The proposed ZVT PWM Cük PFC.

To analyze the steady state operations of the circuit shown in Fig. 1, following assumptions are made during one switching cycle:

(1). Input voltage $V_{s}$ is constant.

(2). Output capacitor $\mathrm{C}_{0}$ is large enough to assume that the output voltage $V_{o}$ is constant and ripple-free.

(3). Inductors $\mathrm{L}_{1}$ and $\mathrm{L}_{2}$ are much greater than resonant inductor $\mathrm{Lr}$.

(4). Capacitor $\mathrm{Cl}_{1}$ is much greater than resonant capacitor $\mathrm{C}$.

(5). All semiconductor devices are ideal, except the main diode $\mathrm{D}_{1}$.

Based on these assumptions, circuit operations during one switching cycle can be divided into seven stages, which are shown in Fig. 2 (a)-(g), respectively.

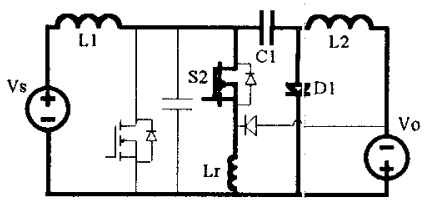

(a) Stage 1

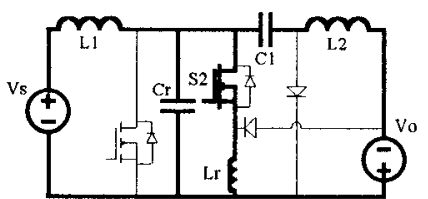

(b) Stage 2

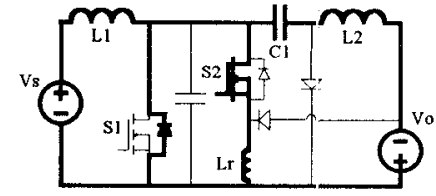

(c) Stage 3

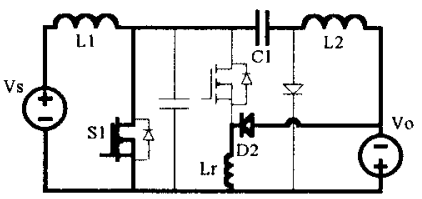

(d) Stage 4

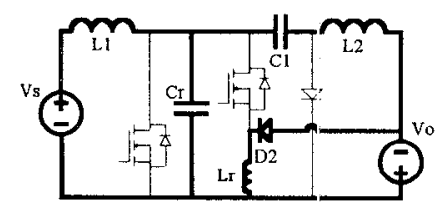

(e) Stage 5

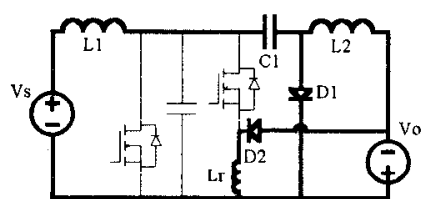

(f) Stage 6

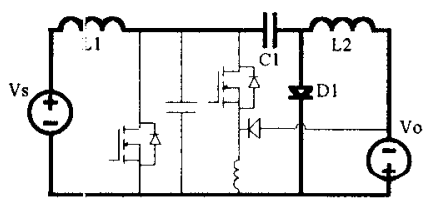

(g) Stage 7

Fig. 2. Equivalent circuits during one switching cycle.
Stage 1 (Fig. $2 . a ; t_{0}<t<t 1$ ):

The auxiliary switch $\mathrm{S}_{2}$ turns on at to. During turnon process of $S_{2}$, injected charges in the low-doped middle region of diode $D_{1}$ cause transient reverse recovery current flowing reversely through diode $\mathrm{D}_{1}$. Growth rate of the reverse recovery current is restricted by the resonant inductor $\mathrm{Lr}$ to achieve ZCS turn-on of the auxiliary switch S2. Switching losses and EMI noises are reduced due to smoother current slope. Assuming that the current of the resonant inductor, ILr, is zero before to, ILr can be given by

$$
I_{L r}(t)=\frac{V_{C 1}}{L_{r}}\left(t-t_{0}\right)
$$

Assuming that trr is the reverse recovery time of $\mathrm{D}_{1}$, peak value of the reverse recovery current, Irr, and time interval of stage 1, to1, can be given by

$$
\begin{aligned}
& I_{r r}=I_{L r}\left(t_{1}\right)-I_{L 1}-I_{L 2} \approx \frac{V_{C 1} t_{r r}}{2 L_{r}} \\
& t_{01}=t_{1}-t_{0} \approx \frac{\left(I_{L 1}+I_{L 2}\right) L_{r}}{V_{C 1}}+\frac{t_{r r}}{2}
\end{aligned}
$$

Stage 2 (Fig. 2.b $\mathrm{t}_{1}<\mathrm{t}<\mathrm{t} 2$ ):

The reverse recovery phenomenon finishes at $t 1$. As soon as $\mathrm{D}_{1}$ is turned off, the resonant capacitor $\mathrm{Cr}$ starts to discharge to $\mathrm{Lr}$ through S2. Growth rate of the voltage across $\mathrm{Dl}_{1}$, which is equal to $\mathrm{VCl}-\mathrm{VCr}$, is restricted by $\mathrm{C}_{r}$ to achieve ZVS turn-off of $\mathrm{D}_{1}$. Assuming that the inductor current IL1 and IL2 are both constant in this stage, resonant inductor current and resonant capacitor voltage are

$$
\begin{aligned}
& I_{L r}(t)=\frac{V_{C 1}}{Z_{1}} \sin \left(\omega_{1}\left(t-t_{1}\right)\right)+I_{r r} \cos \left(\omega_{1}\left(t-t_{1}\right)\right)+I_{L 1}+I_{L 2} \\
& V_{C r}(t)=V_{C 1} \cos \left(\omega_{1}\left(t-t_{1}\right)\right)-I_{r r} Z_{1} \sin \left(\omega_{1}\left(t-t_{1}\right)\right)
\end{aligned}
$$

where

$$
\begin{aligned}
& Z_{1}=\sqrt{\frac{L_{r}}{C_{r}}} \\
& \omega_{1}=\left(L_{r} C_{r}\right)^{-0.5}
\end{aligned}
$$

Time interval of stage $2, \mathrm{t} 12$, can be given by

$$
\begin{aligned}
& t_{12}=t_{2}-t_{1}=\sqrt{L_{r} C_{r}} \sin ^{-1}\left(\sqrt{\frac{C_{r} V_{C 1}^{2}}{L_{r} I_{r r}^{2}+C_{r} V_{C 1}^{2}}}\right) \\
& =\sqrt{L_{r} C_{r}} \cos ^{-1}\left(\sqrt{\frac{L_{r} I_{r r}^{2}}{L_{r} I_{r r}^{2}+C_{r} V_{C 1}^{2}}}\right)
\end{aligned}
$$


Stage 3 (Fig. 2.c; $\mathrm{t}_{2}<\mathrm{t}<\mathrm{t} 3$ ):

When $\mathrm{VCr}$ is discharged to zero at $\mathrm{t}$, body diode of the main switch, Ds1B, is turned on simultaneously. Drain-source voltage of $\mathrm{S} 1$, which equals $\mathrm{VCr}$, retains zero after $\mathrm{t} 2$. ILr retains constant in this stage and it is given by

$I_{L r}(t)=I_{L 1}+I_{L 2}+\sqrt{\frac{L_{r} I_{r r}^{2}+C_{r} V_{C 1}^{2}}{L_{r}}}$

Stage 4 ( Fig. 2.d; $t 3<t<t 4$ ):

The main switch $S_{1}$ turns on and the auxiliary switch S2 turns off simultaneously at $t 3$. The resonant inductor $\mathrm{Lr}$ starts to discharge to the output through the auxiliary diode D2. ILr decreases linearly because the voltage across $\mathrm{Lr}$ is equal to the constant negative output voltage. Since the main switch $S_{1}$ is not on the discharge path of Lr, current through $S_{1}$ raises to the normal turn-on current, which equals the sum of IL1 and IL2, immediately when $S_{1}$ turns on at 13 . In this stage, circuit operation is identical to the turn-on state of a conventional PWM Cúk PFC despite that $\mathrm{L}_{r}$ is discharging to the output.

Stage 5 (Fig. 2.e $; \mathrm{t} 4<\mathrm{t}<\mathrm{ts}$ ):

The main switch $S_{1}$ turns off at $t 4$. After $t 4$, IL1 and IL2 start to charge the resonant capacitor $\mathrm{C}_{\mathrm{r}}$ to achieve ZVS turn-off of $\mathrm{S}_{1}$. Discharge of $\mathrm{Lr}$ can still be executed in this stage even if $S_{1}$ is off. Time interval of stage $5, t 45$, is given by

$$
t_{4 s}=\frac{C_{r} V_{C l}}{I_{t 1}+I_{L 2}}
$$

Stage 6 (Fig. 2.f $; \mathrm{t} 5<\mathrm{t}<\mathrm{t} 6$ ):

The main diode $\mathrm{D}_{1}$ is turned on under $\mathrm{ZVS}$ when $\mathrm{VCr}$ is charged to $\mathrm{VCl}$ at 15 . The resonant inductor $\mathrm{Lr}$ can keep discharging in this stage without influence of the state of $S_{1}$ and $D_{1}$.

Stage 7 ( Fig. 2.g $; \mathrm{t}_{6}<\mathrm{t}<\mathrm{t} 0$ ):

Energy recovery process is finished after $\mathrm{ILr}$ is discharged to zero at t6. Auxiliary diode $\mathrm{D}_{2}$ is also turned off under ZCS at the same time. After that, circuit operation is identical to the turn-off state of a conventional PWM Cúk PFC. It is back to stage 1 when the auxiliary switch $S_{2}$ turns on again at to in the next switching cycle.

Based on the analysis presented above, key waveforms of the proposed ZVT PWM Cúk PFC are shown in Fig. 3.

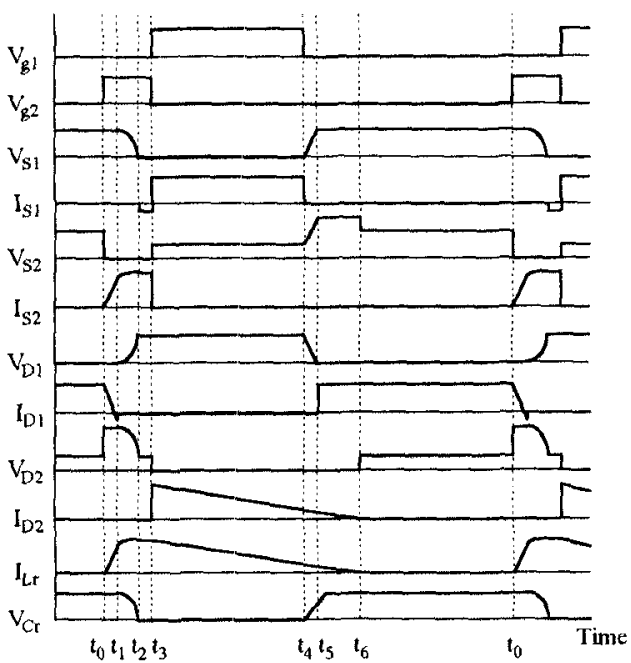

Fig. 3. Key waveforms of the proposed ZVT PWM Cuk PFC.

\section{B. Basic Features of the Circuit}

- Minimum ZVT time

The major difference between the proposed ZVT Cúk topology and the conventional ZVT Cúk topology [6] is the discharge path of the resonant inductor. In the proposed topology, discharge current does not flow through the main switch. Circuit operation becomes the same as the normal on-state of a common Cúk circuit as soon as the main switch turns on. The ZVT time 102 , which equals $12-10$, is comparatively short. In the conventional topology, since the discharge current flows through the main switch, the current of the main switch increases slowly after the main switch turns on. It lengthens the ZVT time. Assuming that $\mathrm{Irr}_{\mathrm{r}}$ is comparatively small, ZVT time of the proposed topology, TZVT1, can be given by

$$
\begin{aligned}
& T_{z r r 1}=t_{02}=t_{01}+t_{12} \\
& \approx \frac{\left(I_{L 1}+I_{L 2}\right) L_{r}}{V_{C 1}}+\frac{t_{r r}}{2}+\frac{\pi}{2} \sqrt{L_{r} C_{r}}
\end{aligned}
$$

and ZVT time of the conventional topology, TZVT2, is given by

$$
\begin{aligned}
& T_{z r r 2} \approx \frac{\left(I_{L 1}+I_{L 2}\right) L_{r}}{V_{C 1}}+\frac{t_{r r}}{2}+\frac{\pi}{2} \sqrt{L_{r} C_{r}}+\frac{I_{L r, p} L_{r}}{V_{C 1}} \\
& =\frac{2\left(I_{L 1}+I_{L 2}\right) L_{r}}{V_{C 1}}+t_{r r}+\frac{\pi}{2} \sqrt{L_{r} C_{r}}+\sqrt{L_{r} C_{r}} \\
& \approx 2 T_{z r r 1}
\end{aligned}
$$

It is shown that ZVT time of the proposed topology is only half of the conventional one. Actually, the proposed ZVT topology has the shortest ZVT time in all of the ZVT topologies which can be applied to a Cúk 
PFC. The voltage and current waveforms of both the main and auxiliary switches are essentially squarewaves except during ultrashort ZVT time. Various control strategies and design rules of PMW techniques are suitable to be applied to the proposed circuit.

- Short minimum duty cycle of main switch

The minimum duty cycle of the main power switch is very important in PFC applications because it establishes how much high the output voltage must be for linear operation. The output voltage during light load is also more stable with shorter minimum duty cycle. In the conventional ZVT PWM Cúk topology [6], the minimum duty cycle is dominated by discharge time of the resonant inductor Lr. In the proposed one, since the discharge current of $\mathrm{Lr}$ does not flow through the main switch $\mathrm{S}_{1}$, discharge of $\mathrm{Lr}$ can be executed even if $\mathrm{S} 1$ is off. In other words, the minimum duty cycle of the main switch can be shorten regardless of discharge time of Lr. Due to the extremely short minimum duty cycle, the proposed circuit has wide output voltage range and wide switching frequency range. It is especially suitable to be employed under variable duty and high switching frequency applications, such as PFC's.

\section{- Constant operating frequency}

Since the proposed ZVT topology is based on PWM technique, the operating frequency is constant. Constant operating frequency makes the design optimization of EMI filter easily attained. Various control strategies designed for PWM technique, such as current mode control, are also applicable for the proposed ZVT Cúk PFC. UC3855 and ML4822, which are current mode control IC's designed for the ZVT PWM topology in [6], can be employed in the proposed circuit as well.

\section{-Long resonant inductor discharge time}

One drawback of the conventional ZVT PWM topology [6] is that the resonant inductor can not be discharged when the main switch turns off. In the conventional topology, the main diode and the auxiliary diode are essentially in parallel. When the main diode is conducting, a certain percentage of current will flow through the auxiliary diode. This undesirable feature causes considerable switching losses when the auxiliary switch turns on unless an additional saturable reactor is placed in series with the resonant inductor.

The situation is opposite in the proposed ZVT topology. In the proposed ZVT PWM Cúk PFC, the resonant inductor can keep discharging even if the main switch turns off. It ensures that the current through the resonant inductor and the auxiliary diode keeps zero after the resonant inductor is completely discharged. The turn-on loss of the auxiliary switch is successfully limited without the additional saturable reactor. Contrarily, since larger value of the resonant inductor can be used due to long discharge duration, the turn-on loss of the auxiliary switch in the proposed ZVT topology is smaller than that of the conventional one. .

\section{-Soft switching for wide line and load ranges}

One drawback of other soft switching techniques is that the soft switching condition is strongly dependent on load current and input voltage. At light load, ZVS is usually difficult to maintain, since the energy stored in the resonant inductor at light load is not sufficient to completely discharge the resonant capacitor prior to turn-on of the active switch. At high line, ZVS is easier to lose, since: it needs more energy to discharge the resonant capacitor. Although losing ZVS at light load or high line does not cause serious thermal problems, EMI due to switching noises may be intolerable in a practical circuit.

In ZVT PWM topologies, soft switching is maintained in wide load and line ranges as long as it has been designed for high load or low line. This desirable feature makes ZVT PWM topologies more suitable to be employed in PFC applications. For wide line and load range, especially in a $\mathrm{PFC}$, wide variation range of duty cycle is necessary. Since the proposed ZVT Cúk topology has the shortest minimum duty cycle, it can be operated with wider line and load ranges compared with other ZVT PWM topologies.

\section{Isolated Single-stage ZVT PWM Cúk PFC}

The proposed ZVT PWM Cúk PFC can be extended to isolated single-stage form as shown in Fig. 4. To ensure complete isolation, the resonant inductor $\mathrm{Lr}$ is replaced by the coupled inductors $\mathrm{Lr}_{1}$ and $\mathrm{Lr}$. In deriving the isolated ZVT topology of Fig. 4, two key characteristics of the nonisolated ZVT topology of Fig. 1 were preserved [12]. First, the ZVT time and the minimum duty cycle are extremely short. Second, discharge current of the coupled resonant inductors $\mathrm{Lr}_{1}$ and $\mathrm{Lr}_{2}$ does not flow through the main switch. Circuit operations are similar to those of non-isolated counterpart.

To avoid large overvoltages across switch $\mathrm{S}_{2}$ at turn-off, a very small leakage inductance and a very high coupling between the primary and the secondary windings should be carefully built when designing the coupled inductors $\operatorname{Lr} 1$ and Lr2. Since good dynamic characteristics are necessary to the diode $\mathrm{D}_{2}$, a fast forward and reverse recovery diode is needed. 


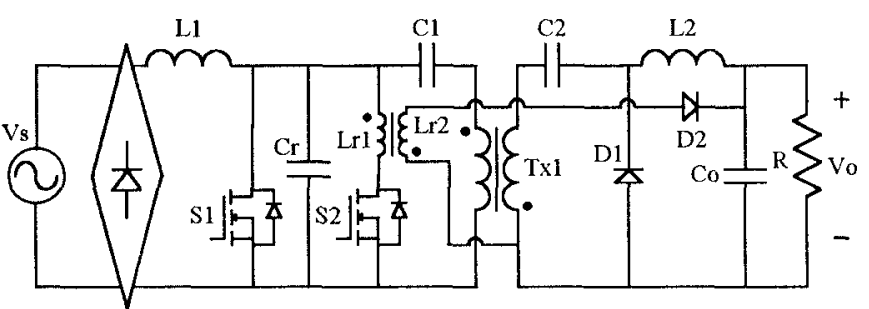

Fig. 4. Isolated single-stage ZVT PWM Cúk PFC.

\section{DESIGN PROCEDURE}

Since design of the conventional Cúk circuit has been well presented in literature, it is more significant to focus on design rules of the proposed ZVT cell. The resonant inductor and the resonant capacitor are the most important components when designing the ZVT cell. The resonant inductor is designed to provide soft turn-on of the main switch and the auxiliary switch while the resonant capacitor is designed to provide soft turn-off of the main switch.

\section{A. Resonant Inductor}

The resonant inductor value can be obtained by determining how fast the main diode can be turned off. However, it is difficult to calculate accurately because of the recovery characteristics variation among different rectifiers. A widely adopted estimate is to allow the current through the resonant inductor to ramp up to the diode current within three times the diode's specified reverse recovery time. One constraint on the maximum inductance value is its affect on the minimum duty cycle [10-11].

The resonant inductor design is limited by core loss instead of saturating flux density due to the high AC component and the relatively high operating frequency. Resonant ringing at turn-off of the auxiliary switch is easily generated by high loss material resonant inductor. High frequency response and low loss core materials such as molly-permalloy powder (MPP) are adequate. The resonant inductor peak current can be approximated by

$$
I_{L r, p}=I_{L 1}+I_{L 2}+\frac{V_{C 1}\left(t_{r r}+2 \sqrt{L_{r} C_{r}}\right)}{2 L_{r}}
$$

it determines the radius of winding wires.

\section{B. Resonant Capacitor}

The resonant capacitor is designed to control $\mathrm{dv} / \mathrm{dt}$ of the drain-source voltage of the main switch. It provides an alternative path for the inductor current when the main switch turns off to reduce switching losses and dv/dt EMI noises. High frequency response capacitors with low ESR and ESL, such as polypropylene film, are required. A good estimate is to determine the total capacitor value, Ctotal, by setting the turn-off transient time, $t 45$.

$$
C_{\text {total }}=\frac{\left(I_{L 1}+I_{L 2}\right) t_{45}}{V_{C 1}}
$$

Since the resonant capacitor incorporates the output capacitance of the main switch and the main diode, the resonant capacitor value can be obtained by subtracting output capacitor value of the main switch and main diode from the total capacitor value Ctotal.

\section{Auxiliary Switch and Diode}

The voltage stresses of the auxiliary switch and diode are equal to $\mathrm{VCl}+\mathrm{V}_{\mathrm{o}}$. The current stresses of the auxiliary switch and diode are equal to the peak current of the resonant inductor as shown in (13). Since the short duty cycle of the auxiliary switch makes the conduction loss comparatively low, a MOSFET smaller than the main switch is sufficient. The auxiliary diode needs to be an fast recovery diode to prevent the resonant inductor from resonating with the output capacitor after discharge.

\section{EXPERIMENTAL RESULTS}

Prototype of a $400 \mathrm{~W}, 50 \mathrm{kHz},-200 \mathrm{~V} \mathrm{DC}$ output ZVT PWM Cúk PFC, as shown in Fig. 1, has been built to verify the principle of operation and the theoretical analysis. The components specifications are listed in Table 1.

TABLE 1

PART LIST OF THE IMPLEMENTED PROTOTYPE POWER CIRCUIT

\begin{tabular}{|c|c|c|c|c|c|}
\hline Part & Type & Part & Type/Value & Part & Value \\
\hline S1 & 2 SK2198 & $\mathrm{D}_{2}$ & HFA15TB60 & $\mathrm{C}_{1}$ & $4.7 u \mathrm{~F}$ \\
\hline $\mathrm{S}_{2}$ & IRFP450 & $\mathrm{L}_{1}, \mathrm{~L} 2$ & $800 u \mathrm{H}$ & $\mathrm{Lr}$ & $30 u \mathrm{H}$ \\
\hline $\mathrm{D}_{1}$ & HFA25TB60 & $\mathrm{Co}_{\mathrm{r}}$ & $660 u \mathrm{~F}$ & $\mathrm{Cr}_{\mathrm{r}}$ & $3.3 \mathrm{nF}$ \\
\hline
\end{tabular}

Efficiency of $95.2 \%$ has been measured at full load. Key oscillograms of the proposed ZVT PWM Cúk PFC are shown in Fig. 5. It can be seen that the waveforms agree well with the predicted ones shown in Fig. 3. The' operation analysis is guaranteed to be valid. Fig. 5(a) shows that the main switch $S_{1}$ commutates under ZVS turn-on and turn-off since the non-ideal commutation phenomena are eliminated by the shunt resonant ZVT cell. It can also be seen from Fig. 5 (c) that the main diode $\mathrm{D}_{1}$ is also commutated under ZVS turn-on and turn-off. Soft switching applies to not only the 
main switch $\mathrm{S}_{1}$ but also the main diode D1. Fig. 5(b) shows that the auxiliary switch $S_{2}$ commutates near ZCS turn-on while Fig. 5(d) shows that the auxiliary diode $D_{2}$ is commutated under ZCS turn-off. The reason that $\mathrm{S}_{2}$ can only commutate near ZCS turn-on is because the discharge of parasitic drain-source capacitance. However, since the parasitic capacitance of $S_{2}$ is smaller than that of $S_{1}$ and the reverse recovery current of $D_{1}$ is restricted by $L_{r}$, only few switching loss is generated when $\mathrm{S}_{2}$ turns on. Current of $\mathrm{Lr}_{r}$ and voltage of $\mathrm{Cr}_{r}$ are shown in Fig. 5(e).

A comparatively large inductor value of $\mathrm{Lr}$ is used to emphasize the desirable peculiarity of the proposed ZVT topology. The large Lr effectively decreases the turn-on loss and the current stress of $S_{2}$ while keeping the ZVT time still short. It can be seen from Fig. 5(b) and Fig 5(d) that the auxiliary switch $S_{2}$ and the auxiliary diode $D_{2}$ are activated for short ZVT time duration. The ZVT PWM converters are identical to common PWM converters during rest of the time. Control strategies and design rules of common PWM converters can be directly applied to ZVT PWM converters. Current ratings of ausiliary semiconductor devices can also be reduced due to short $Z V T$ time.

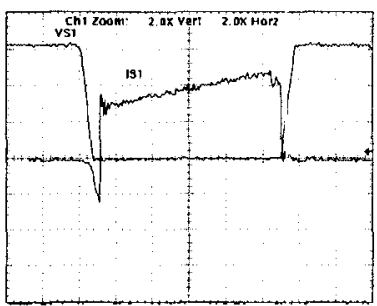

(a) VSI and IsI

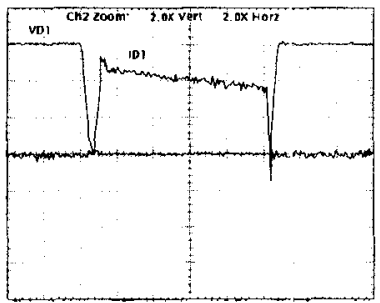

(c) VDl and ID

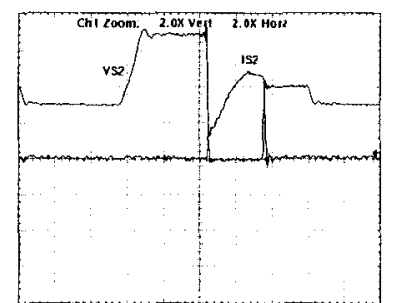

(b) V S2 and Is2

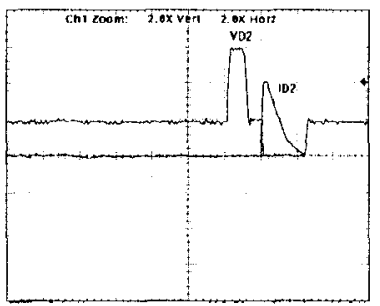

(d) VD2 and ID2

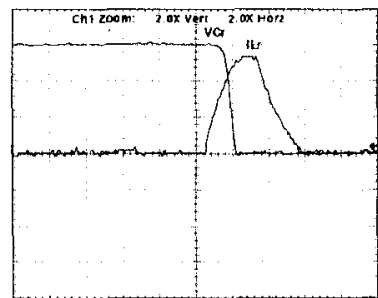

(e) $\mathrm{VCr}$ and $\mathrm{ILr}$

Fig. 5. Key oscillograms of the proposed ZVT PWM Cúk PFC Vs1, Vs2, VDI, VCr : 100V/div; VD2 : 200V/div;

IS1,IS2,ID1,ID2,ILr : 4A/div; Time $2 u$ s/div.

\section{CONCLUSION}

A novel ZVT PWM Cúk PFC is proposed in this paper Ideally, no switching loss is generated from the main switch and the main diode. Constant switching frequency eases the design of EMI filters and control circuits. Wide line and load ranges are also achieved since ZVS and ZCS are easily maintained at not only low line and heavy load but also high line and light load. Advantages of both ZVT converters and Cúk PFC's are obtained in the proposed circuit. Besides, to apply ZVT techniques to PFC topologies more appropriately, the proposed ZVT technique has an extremely short ZVT time. Discharge of the resonant inductor is also robuster and more reliable than the conventional ZVT technique. Prototype of a $400 \mathrm{~W}, 50 \mathrm{kHz}$ ZVT PWM Cúk PFC is built in laboratory to experimentally verify the analysis. It can be shown from the oscillograms that soft switching is successfully applied to the ZVT PWM Cúk PFC.

\section{REFERENCES}

[1] B. R. Lin and T. S. Huang, "Analysis of ZCS Cuk converter for power factor correction," ISIE'96, pp. 755-760.

[2] R. Antonio, D. Abud, G. Jimenez and J. Arau, "Evaluation of boost, SEPIC and Cuk topologies as power factor correction stage in electronic ballast applications," CIEP'94, pp. \$1-55.

[3] N. Mohan, T. Undeland and W. Robbins, Power Electronics: Converters, Applications and Design. Wiley, 1989, pp. 98-99.

[4] M. Ferranti, P. Ferraris, A. Fratta and F. Profumo, "Solar energy supply system for induction motors and various loads," INTELEC'89, paper 15.7.

[5] G. A. Karvelis and S. N. Manias, "Fixed-frequency buck-boost zerovoltage-switched quasiresonant converter," IEE Proc. Electr. Power Appl., vol. 142, no. 5, pp. 289-296, Sep. 1995.

[6] G. Hua, C. S. Leu, Y. Jiang and F. C. Lee, "Novel zero-voltagetransition PWM converters," IEEE Trans. Power Electron., vol. 9, no. 2, pp. 213-219, Mar. 1994.

[7] G. Moschopoulos, P. Jain and G. Joos, "A novel zero-voltage- switched PWM boost converter," PESC'95, pp. 694-700.

[8] G. Moschopoulos, P. Jain, Y. F. Liu and G. Joos, "A zero-voltageswitched PWM boost converter with an energy feedforward auxiliary circuit," PESC'96, pp. 76-82.

[9] G. Hua and F. C. Lee, "Soft-switching techniques in PWM converters," IEEE Trans. Ind. Electron., vol. 42, no, 6, pp. 595-603, Dec. 1995.

[10] J. Bazinet and J. A. O'Connor, "Analysis and design of a zero voltage transition power factor correction circuit," APEC'94, pp. 591-597.

[11] J. P. Noon, "A $250 \mathrm{kHz}, 500 \mathrm{~W}$ Power Factor Correction Circuit Employing Zero Voltage Transitions," Unitrode Power Supply Design Seminar Manual SEM1000, Unitrode Corp., Waltham,. MA, pp. 1.1$1.15,1994$

[12] A. Elasser and D. A. Torry, "Soft switching active snubbers for $\mathrm{dc} / \mathrm{dc}$ converters," IEEE Trans. Power Electron., vol. 11, no. 5, pp. 710-722, Sep. 1996. 